\title{
Photonic band gap in a superconductor-dielectric superlattice
}

\author{
C. H. Raymond Ooi, T. C. Au Yeung, C. H. Kam, and T. K. Lim \\ School of EEE, Nanyang Technological University, Singapore \\ (Received 1 June 1999; revised manuscript received 10 August 1999)
}

\begin{abstract}
We foresee applications and interesting possibilities of incorporating the photonic crystals concept into superconducting electronics. In this paper, we present interesting features of the computed lower band structure of a nondissipative superconductor-dielectric superlattice using the two-fluid model and the transcendental equation [Pochi Yeh, Optical Waves in Layered Media, Wiley Series in Pure and Applied Optics (Wiley, New York, 1988)]. The necessary conditions for approximating the complex conductivity by an imaginary conductivity is derived and the feasibility of achieving the conditions are discussed. The superlattice dispersion obtained is similar to that of the phonon-polariton dispersion in ionic crystal. We found a nonlinear temperature-dependent "polariton gap" and a low-frequency (plasma) gap, and suggested the existence of a photon-superelectron hybrid around the polariton gap. The polariton gap may be observed in an infraredmicrowave regime using a high- $T_{c}$ superconductor with sufficiently low normal-fluid relaxation time $\left(\approx 10^{-15} \mathrm{~s}\right)$, and in an optical regime using lower penetration depth $(\approx 50 \mathrm{~nm})$ and extremely low relaxation time $\left(\approx 10^{-17} \mathrm{~s}\right)$.
\end{abstract}

I. Introduction. Much work has been done on the computation of the band structures of electromagnetic waves propagating in two- and three-dimensional dielectric periodic structures since it was shown ${ }^{1}$ that these periodic structures can be designed to produce the required band structures. The band structures explored were mainly fabricated from dielectric materials, ${ }^{2-4}$ typically used in the semiconductor technology. Dielectric periodic structures can be designed to mold the light propagation in integrated semiconductor optoelectronics where electronic and optical signals coexist and transform between each other.

Recently, combinations of various materials for the design of photonic crystals have been studied. Sigalas et al..$^{5}$ found wider photonic band gaps when dielectric constant $\varepsilon$ and relative permeability $\mu$ have their maximum values in different materials and suggested using magnetically tuned ferrite materials. Electric- and magnetic-field-dependent materials like ferroelectrics, ferromagnets, and ferrimagnets were investigated in two-dimensional photonic crystals. ${ }^{6}$ Frequencydependent dielectrics ${ }^{7}$ and metallic ${ }^{8}$ photonic crystals have been studied, too. We foresee novel applications and interesting possibilities of incorporating the photonic crystals concept into superconducting devices. From this motivation, in this paper we study the band structure of a onedimensional nondissipative superconductor-dielectric superlattice.

We describe the electromagnetic response of a typical nonmagnetic superconductor using the two-fluid model ${ }^{9,10}$ via the complex conductivity. The necessary and sufficient conditions that reduce the complex conductivity to imaginary conductivity are derived, since we are interested in a nondissipative superlattice. The superconductor satisfies the GorterCasimir relation. ${ }^{11}$ The dielectric layer is characterized by a real dielectric constant in the frequency regime of interest. We apply the source free Maxwell's equations and the wellknown transcendental equation ${ }^{12}$ to compute the band structure for the dielectric-superconducting superlattice. We observe the dispersion curve splitting similar to the phononpolariton dispersion in bulk dielectric, which we refer as the superpolariton (SP) gap and also the low-frequency (LF) gap similar to the plasma frequency gap in alkali metals. The distinct results from this new material structure compared to the all-dielectric superlattice are discussed.

II. Theory. The two-fluid model ${ }^{9}$ is used to describe the ac electrodynamics of a superconductor at nonzero temperature. This model has been proven successful in describing the performance of high-frequency superconductive devices. ${ }^{10,11}$ According to the two-fluid model, the complex electrical conductivity $\sigma=\sigma_{n}+\sigma_{s}$ of a superconductor in the presence of a time harmonic electromagnetic field is due to the unpaired-normal electrons $\sigma_{n}$ and the paired superelectrons $\sigma_{s}$ of density $n_{n}$ and $n_{s}$, respectively, where $n=n_{s}+n_{n}$ is the total density of electrons. By taking the relaxation time $\tau$ to infinity for superconductor and using particle dynamics for both normal electrons and superelectrons, we have the complex conductivity as given in Ref. 9,

$$
\begin{aligned}
\sigma= & \left(e^{2} / m\right)\left[\left\{n_{n} \tau /\left(1+\tau^{2} \omega^{2}\right)+\delta(\omega) \pi n_{s} / 2\right\}\right. \\
& \left.-j\left\{\tau^{2} \omega n_{n} /\left(1+\tau^{2} \omega^{2}\right)+n_{s} / \omega\right\}\right] .
\end{aligned}
$$

For nonzero frequency, Eq. (1) reduces to

$$
\sigma=\left(e^{2} / m\right)\left[n_{n} \tau(1-j \tau \omega) /\left(1+\tau^{2} \omega^{2}\right)-j n_{s} / \omega\right] .
$$

In order to find the condition that enables for imaginary conductivity approximation, we first assume that $\tau^{2} \omega^{2} \ll 1$. If we set 0.01 as sufficiently much less than 1 , we have $\omega \leqslant 0.1 / \tau$ (Condition 1). At a fixed temperature, $\tau(T)$ is fixed, and therefore we restrict our study to the low-frequency regime. Here, Eq. (2) reduces to

$$
\sigma(\omega)=\left(e^{2} / m\right)\left[n_{n} \tau(1-j \tau \omega)-j n_{s} / \omega\right] .
$$

When $n_{n} \tau \ll j n_{s} / \omega$ is satisfied, the complex conductivity [Eq. (3)] approximates to imaginary

$$
\sigma(\omega) \cong-j e^{2} n_{s} / m \omega .
$$

Again, using the 0.01 limit, we obtain $\omega \tau \leqslant 0.01 n_{s} / n_{n}$ (Condition 2). 
Condition 1 and Condition 2 enable the conductivity to be simply expressed in terms of the London penetration depth $\lambda_{L}$, since $m / \mu_{0} n_{s} e^{2}=\lambda_{L}^{2}$,

$$
\sigma(\omega) \cong-j / \mu_{o} \omega \lambda_{L}^{2}
$$

From the Gorter-Casimir result ${ }^{11} n_{s} / n_{n}=\left(T_{c} / T\right)^{4}-1$ and the London penetration depth, we obtain

$$
\lambda_{L}(T)=\lambda_{L}(0) / \sqrt{1-\left(T / T_{c}\right)^{4}},
$$

where the conductivity [Eq. (5)] is temperature dependent.

Combining Condition 1 and Condition 2 and using Eq. (6), we have

$$
\begin{gathered}
\tau \omega \leqslant \text { minimum }\left(0.01\left[\left(T_{c} / T\right)^{4}-1\right], 0.1\right), \\
\tau \omega \leqslant \text { minimum }\left(0.01 /\left[\left(\lambda_{L} / \lambda_{L o}\right)^{2}-1\right], 0.1\right) .
\end{gathered}
$$

For the temperature range of $0.01\left[\left(T_{c} / T\right)^{4}-1\right] \geqslant 0.1$ or $T$ $\leqslant 0.5491 T_{c}$, the frequency range must satisfy $\tau \omega \leqslant 0.1$, while for temperature range of $T_{c} \geqslant T \geqslant 0.5491 T_{c}$, the condition $\tau \omega \leqslant 0.01\left[\left(T_{c} / T\right)^{4}-1\right]$ must be satisfied for the imaginary conductivity approximation to hold.

We consider a superlattice with period ' $a$,', composed of alternating superconducting layers and a dielectric layer of thickness " $d$,' with each layer in the $y-z$ plane.

By using Eq. (5) in Maxwell's equations, the dispersion for the superconducting layer can be written as

$$
\begin{gathered}
k_{s}^{2}=(\omega / c)^{2}-\left(1 / \lambda_{L}\right)^{2}, \\
k_{s x}^{2}=(\omega / c)^{2} \cos ^{2} \theta-\left(1 / \lambda_{L}\right)^{2},
\end{gathered}
$$

where Eq. (8b) follows from the continuity of the fields along the $y-z$ plane and $\theta$ is the angle of incidence relative to normal of interfaces.

The transfer-matrix method ${ }^{12}$ gives the transcendental equation for a lossless superlattice

$$
\begin{aligned}
\cos k_{B} a= & \cos \left[k_{s x}(a-d)\right] \cos \left(k_{x} d\right)-\frac{1}{2}(p / q+q / p) \\
& \times \sin \left[k_{s x}(a-d)\right] \sin \left(k_{x} d\right),
\end{aligned}
$$

where $k_{B}$ is the Bloch wave vector and $k_{x}$ is the normal component of the wave vector $k$ in the dielectric layer.

For $E(H)$ polarization, the electric field $E(H)$ is along the $y-z$ plane and we have

$$
\begin{gathered}
(p / q)_{e}=\frac{k_{x}}{k_{s x}}=\sqrt{\frac{\varepsilon-\sin ^{2} \theta}{\cos ^{2} \theta-\left(c / \omega \lambda_{L}\right)^{2}}} \\
(p / q)_{h}=\frac{k_{x} k_{s}^{2}}{k_{s x} k^{2}}=\sqrt{\frac{\varepsilon-\sin ^{2} \theta}{\cos ^{2} \theta-\left(c / \omega \lambda_{L}\right)^{2}}} \frac{1-\left(c / \omega \lambda_{L}\right)^{2}}{\varepsilon} .
\end{gathered}
$$

The right-hand side of Eq. (9) is always real for real $\varepsilon$, even though $k_{s x}$ may be imaginary. The points at $k_{B}=0$ and $k_{B}=\pi / a$ correspond to the band-gap edges and are used to locate the frequency position of the polariton band gap and low-frequency band-gap edges. The plotted frequency $\omega / c$, Bloch wave vector $k_{B}$, and inverse London penetration depth $1 / \lambda_{L}$ are all normalized in units of $2 \pi / a$. The band structure is computed using Eq. (9) with four predetermined

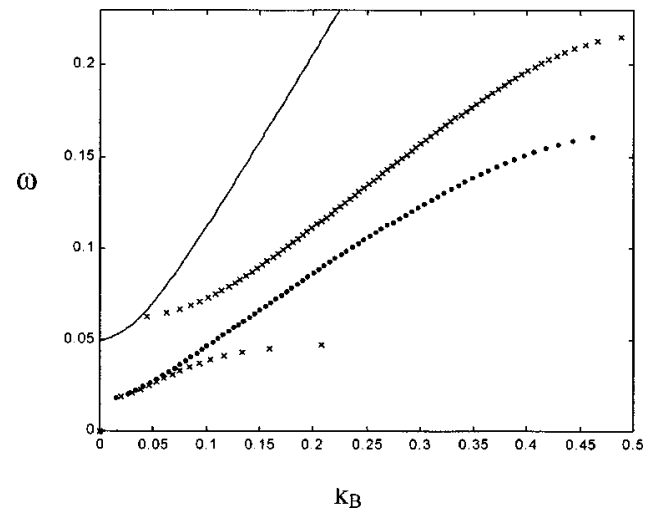

FIG. 1. Superpolariton (SP) gap and low-frequency (LF) gap for $E$ polarization $(\mathbf{O}), H$ polarization $(\times)$ and bulk superconductor (solid line) at incidence angle $\theta=45^{\circ}, 1 / \lambda_{L}=0.05, \varepsilon=15$, and $r$ $=\frac{1}{3}$.

parameters: penetration depth $\lambda_{L}$, angle of incidence $\theta$, dielectric thickness ratio $r=d / a$, and dielectric constant $\varepsilon$.

III. Results and discussion. Two band gaps are observed in the vicinity of $\omega_{\lambda}=c / \lambda_{L}$ (Fig. 1). The low-frequency (LF) band gap $\left(\omega_{1}\right)$ ranges from zero frequency to a threshold designated as $\omega_{1}$. The LF gap is due to the combined effect of both superconducting material and the periodicity since $\omega_{1}$ does not coincide exactly with $c / \lambda_{L}$ (as for bulk superconductor) and the all-dielectric superlattice has no gap ranging from zero frequency. Another band gap $\left(\omega_{2}-\varpi\right)$ ranges from frequency near $c / \lambda_{L}$, designated as $\varpi$ to the next threshold designated as $\omega_{2}$ (Fig. 2). The splitting of the lowest dispersion branch is similar to the phonon-polariton dispersion curve for bulk dielectric. ${ }^{14}$ So, in the frequency around $\omega_{\lambda}$, the dispersion property of the whole superlattice is similar to a bulk ionic crystal. The frequency thresholds $\omega_{2}$ and $\varpi$ are analogous to the longitudinal and transverse optical phonons, respectively. ${ }^{15}$ For convenience, we refer to the $\left(\omega_{2}-\varpi\right)$ band gap as a superpolarition (SP) gap. At frequencies near $\varpi, \omega_{1}$, and $\omega_{2}$, the dispersion is superelectronlike. At a frequency around the SP gap the normal component electric field couples strongly with the superelectrons to form the photon-superelectron hybrids (superpolaritons). At frequencies well above $\omega_{2}$, the dispersion becomes photonlike and the whole superlattice can be represented by an effective dielectric constant, $\varepsilon d / a$, characterized by a translated linear electromagnetic wave dispersion (Fig. 2),

$\omega-\omega_{1}=k_{B} c / \sqrt{\varepsilon d / a}$.

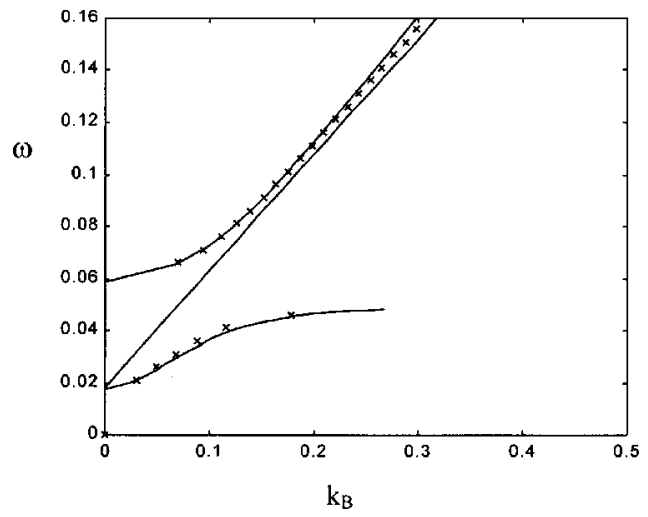

FIG. 2. Fitting of $H$-polarization dispersion curves around the superpolariton gap using Eq. (12) (solid lines), with $\omega_{2}=0.061$, $\varpi=0.05$, and $\omega_{1}=0.018$. The straight line is plotted from Eq. (11). 


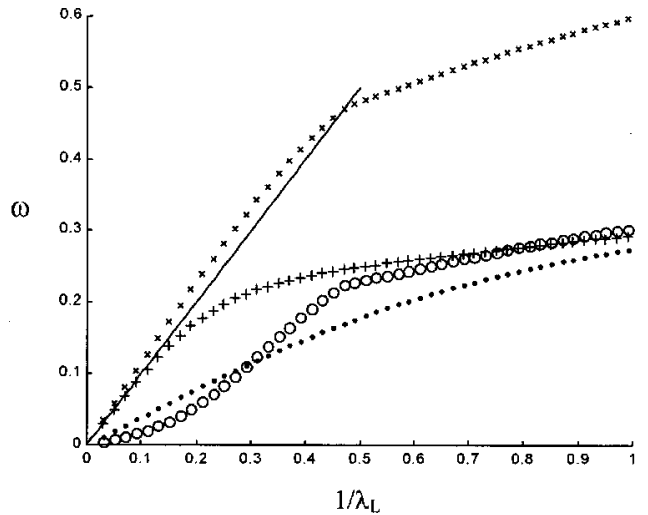

FIG. 3. SP gap (○), LF gap $(\bigcirc), \omega_{2}(+)$, and $\varpi(\times)$ versus $1 / \lambda_{L}$, with $\theta=45^{\circ}, \varepsilon=5$, and $r=0.5$. The straight line serves as a reference, with $\omega=c / \lambda_{L}$.

At a frequency below $\omega_{\lambda}$, even though the wave vector in the superconducting layer is purely imaginary, propagating modes still exist in the superlattice (Fig. 1) with dispersion characterized by the lower superpolariton branch. Here, the electromagnetic energy is not lost but transferred into superelectron oscillations via strong photon-superelectron coupling.

Figure 1 shows that the splitting is solely due to the coupling between the superelectrons and the normal component of electric field $E_{x}$, which is nonzero only for $H$ polarization at oblique angle of incidence $(\theta \neq 0)$. The splitting is not purely due to Bragg reflection but is uniquely due to both the periodicity of the superlattice and the superconducting material, since dielectric superlattices do not have such a gap ${ }^{16}$ and Fig. 1 shows that the SP gap does not exist in bulk superconductor. In contrast, the phonon-polariton gap arises for reasons not due to periodicity of atomic lattice. ${ }^{17} \mathrm{We}$ found an analytical dispersion relation that fits well at the frequency around $c / \lambda_{L}$ (Fig. 2),

$$
\begin{aligned}
\left(k_{B} c\right)^{2} & \approx A \varepsilon(d / a)\left(\omega_{2}^{2}-\omega^{2}\right)\left(\omega^{2}-\omega_{1}^{2}\right) /\left(\varpi^{2}-\omega^{2}\right) \\
& \equiv \omega^{2} \varepsilon_{e}(\omega)
\end{aligned}
$$

where $A=1$ for the lower branch and $A=\left(1-\omega_{1} / \omega\right)^{2}$ for the upper branch, and $\varepsilon_{e}(\omega)$ is the equivalent dielectric function.

The gap sizes increase with the decrease in penetration depth (Fig. 3). The less the fields can penetrate into the superconducting layer, the more the fields are concentrated in the dielectric layer. The greater the difference in field distribution between the dielectric layer and the superconducting layer, the larger the gap splitting, because it leads to greater contrast of electromagnetic field distribution between the upper and lower frequencies of a band gap. As $1 / \lambda_{L}$ exceeds 0.47 , the field penetration becomes sufficiently smaller that the SP effect vanishes. The photon-superelectrons coupling is less extensive and confined around the layer interfaces. The upper polariton branch vanishes and transform into a Bragg dispersion branch for $\omega_{2} \leqslant 1 / \lambda_{L}$. We see that $\omega_{2}$ $>1 / \lambda_{L}>\varpi$ when the SP effect exists, while $\varpi \approx 1 / \lambda_{L}$ in the limit of sufficiently small $1 / \lambda_{L}$ (below 0.01) (Fig. 3). The LF gap seems independent of polarization (Fig. 1) and the angle of incidence $\theta$ (Fig. 4). The dependency of the LF gap on $\theta$

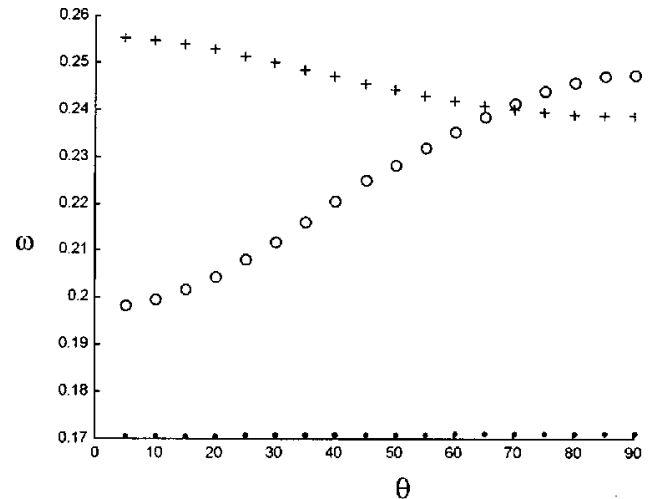

FIG. 4. Superpolariton gap (○), low-frequency gap $(\bullet)$, and $\varpi$ $(+)$ versus angle of incidence $\theta$ with $1 / \lambda_{L}=0.47, \varepsilon=5$, and $r$ $=0.5$.

only becomes obvious for large $1 / \lambda_{L}$. The approximate analytical expression for the LF gap size as a function of $\lambda_{L}, \varepsilon$, and $r$ has been found as

$$
\omega_{1}=A \sin ^{-1}\left(1 / \lambda_{L}\right) \cos ^{-1}(r) / \sqrt{\varepsilon} .
$$

In Figs. (4)-(6) the gaps are plotted with the superpolariton cutoff value of $\left(1 / \lambda_{L c}\right)=0.47$, estimated from Fig. 3 . The monotonic variations of $\omega_{2}$ and SP gap size with $\lambda_{L}, \theta, \varepsilon$, and $r$ (Figs. 3-6) terminate at the "kinks.' These points mark the upper SP effect limits at $\left(1 / \lambda_{L c}\right)=0.47$ (Fig. 3), $\varepsilon_{c}=5$ (Fig. 5), and $r_{c}=0.5$ (Fig. 6), and the lower limits at $\theta_{c}=45^{\circ}$ (Fig. 4). For $\lambda_{L}<\lambda_{L c}, \theta<\theta_{c}, \varepsilon>\varepsilon_{c}$, and $r>r_{c}$, the SP effect vanishes and the gap $\omega_{2}-\varpi$ defines the normal gap instead of the SP gap. The SP gap size is maximum for grazing angle $\theta=90^{\circ}$ and when the normal component electric field $E_{n}$ is maximum (Fig. 4). This supports the explanation that the existence of the SP gap is due to normalelectric-field-superelectron coupling. At $\theta>\theta_{c}$, the normal component electric field is sufficiently stronger for photonsuperelectrons coupling, leading to the band splitting. The SP gap increases with $\varepsilon$ (Fig. 5). At sufficiently high $\varepsilon$ $\left(>\varepsilon_{c}\right)$, the fields in the dielectric layer become highly concentrated at the expense of the fields in the superconducting layer. The weak photon-superelectron coupling leads to the vanishing SP effect. At the limit of bulk superconductor ( $r$ $=0)$, the LF gap approaches $1 / \lambda_{L}$, while at the limit of bulk dielectric $(r=1)$ the LF gap vanishes to zero (Fig. 6).

The frequency within the LF gap and SP gap are approximately below $c / 2 \lambda_{L}$ and around $c / \lambda_{L}$ (Fig. 3). So, the SP effect may be observable for the frequency range around $\omega$

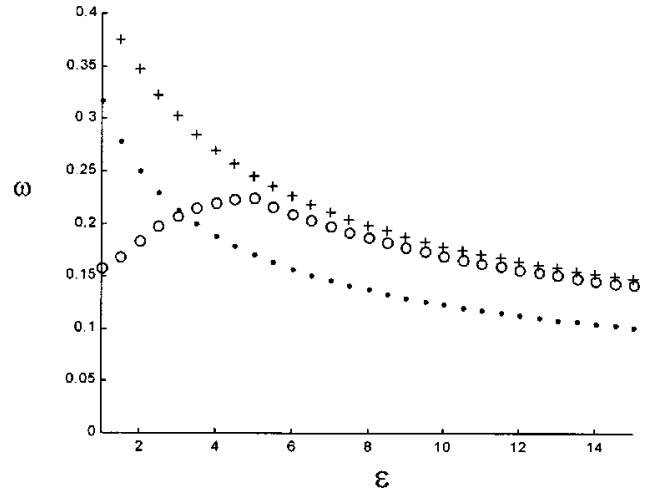

FIG. 5. Superpolariton gap $(\bigcirc)$, low-frequency gap $(\bullet)$, and $\varpi$ $(+)$ versus dielectric constant $\varepsilon$ with $1 / \lambda_{L}=0.47, \theta=45^{\circ}$, and $r$ $=0.5$. 


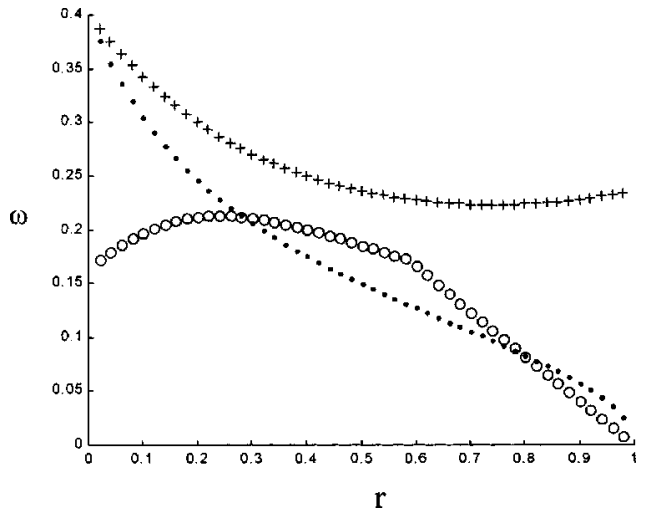

FIG. 6. Superpolariton gap $(\bigcirc)$, low-frequency gap $(\mathbf{O})$, and $\varpi$ $(+)$ versus layer thickness ratio $r$ with $1 / \lambda_{L}=0.47, \theta=45^{\circ}$, and $\varepsilon$ $=5$.

$\approx c / \lambda_{L}$. From Eq. (7b), we have (a) $x^{2}-0.01 x s-1 \leqslant 0$ for $x>1.0488$ or $T_{c} \geqslant T \geqslant 0.5491 T_{c}$, which implies that $s$ $\geqslant 9.5$, (b) $x \geqslant 1 / 0.1 s$ for $x<1.0488$ or $T \leqslant 0.5491 T_{c}$, which implies that $s \geqslant 9.5$, too, where $1<\left(x=\lambda_{L} / \lambda_{L o}\right)<\infty, 0<(t$ $\left.=T / T_{c}\right)<1$, and $s=\lambda_{L o} / c \tau$.

Therefore, the condition $\lambda_{L o} / \tau \geqslant 3 \times 10^{9} \mathrm{~ms}^{-1}$ is required to observe the SP effect in an essentially lossless superlattice. The condition is not a stringent one. Even if the condition is not strictly satisfied but reasonably close to satisfied, we can expect to observe the SP dispersion characteristics close to the results presented in our model. However, our model applies best when $\lambda_{L o} / \tau$ is well beyond $3 \times 10^{9} \mathrm{~ms}^{-1}$. This requirement is most probably satisfied for high- $T_{c}$ superconductors (HTSC's) that have 0-K penetration depth as high as $\approx 1.0 \mu \mathrm{m}$ (Ref. 13) $\left(\mathrm{BaPb}_{0.75} \mathrm{Bi}_{0.25} \mathrm{O}_{3}\right.$ compound). The required relaxation time for the normal electrons at below $T_{c}$ is less than a maximum allowable value $\tau_{\max } \approx 10^{-15} \mathrm{~s}$. This is the typical value for most solid materials at temperatures beyond $100 \mathrm{~K}^{13}$ Therefore, it can most probably be satisfied by HTSC's operating at temperature $T_{\text {op }}$ between $T\left(\tau_{\max }\right)$ and $T_{c}$, in the highly nonlinear regime of $\lambda(T)$, corresponding to large variations in the gaps sizes for a small change in $T_{\text {op }}$ (Fig. 7). Having determined the specific HTSC material to be used, we can now decide on the dimension of the lattice period " $a$ " from the abscissa of Fig. 3 and the SP gap $\left(\omega_{2}\right.$

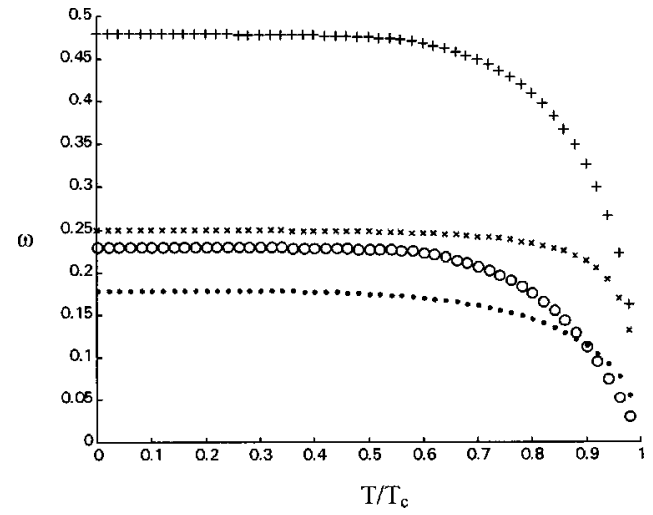

FIG. 7. Superpolariton gap $(\bigcirc)$, low-frequency gap $(\bullet), \omega_{2}$ $(+)$, and $\varpi(\times)$ versus $T / T_{c}$, with $\theta=45^{\circ}, \varepsilon=5$, and $r=0.5$.

$-\varpi)$. For example, at $T_{\mathrm{op}}=0.93 T_{c}, \quad\left[\lambda_{L}(T)=2 \lambda_{L o}\right.$ $=2 \mu \mathrm{m}]$ the abscissa of 0.4 in Fig. 3 corresponds to $\approx 5 \mu \mathrm{m}$ and the SP gap $\left(\omega_{2}-\varpi\right) / 2 \pi=12 \mathrm{THz}$ (infrared microwave). The operating frequency and the SP gap size are in the same order and are mainly determined by the penetration depth via $\omega \approx c / \lambda_{L}$. Therefore, in order to operate at higher frequencies, say $10^{15} \mathrm{~Hz}(300 \mathrm{~nm}=$ optical regime $)$, the penetration depth has to be as small as $50 \mathrm{~nm}$. For this, materials with extremely low normal-fluid relaxation time $\tau\left(<10^{-17} \mathrm{~s}\right)$ are needed. Thus, $\lambda_{L o}$ and $\tau$ are the critical parameters and it is not the SP threshold that determines the operating frequency regime and the feasibility of using existing superconducting, since it is always possible to choose the lattice ratio, dielectric constant, or incident angle, which give the SP effect.

$I V$. Conclusion. In summary, we have discussed basic properties of the dispersion, polaritonlike gap and lowerfrequency gap of a dielectric-superconductor superlattice. We find the required condition $\lambda_{L o} / \tau \geqslant 3 \times 10^{9}$ to observe the SP effect as discussed in our model. The SP effect may be observed in microwave/far-infrared regime using HTSC material that satisfies the Gorter-Casimir relation at temperature extremely close to $T_{c}$. The highly nonlinear temperature dependence of the gap may be useful for temperature sensitive devices. The SP effect may also be observed in the optical regime if the superconducting layer has an extremely low relaxation time.
${ }^{1}$ E. Yablonovitch, Phys. Rev. Lett. 58, 2059 (1987).

${ }^{2}$ K. M. Leung and Y. F. Liu, Phys. Rev. Lett. 65, 2646 (1990).

${ }^{3}$ A. A. Maradudin and M. Plihal, Phys. Rev. B 44, 8565 (1991).

${ }^{4}$ R. D. Meade et al., Phys. Rev. B 44, 13772 (1991).

${ }^{5}$ M. M. Sigalas et al., Phys. Rev. B 56, 959 (1997).

${ }^{6}$ Alex Figotin et al., Phys. Rev. B 57, 2841 (1998).

${ }^{7}$ V. Kuzmaik et al., Phys. Rev. B 55, 4298 (1997).

${ }^{8}$ V. Kuzmaik and A. A. Maradudin, Phys. Rev. B 55, 7427 (1997).

${ }^{9}$ Michael Tinkham, Introduction to Superconductivity, 2nd ed. (McGraw Hill, New York, 1996).

${ }^{10}$ T. van Duzer and C. W. Turner, Principles of Superconductive Devices and Circuits (Arnold, London, 1981), pp. 125-128.

${ }^{11}$ T. van Duzer and C. W. Turner, Principles of Superconductive
Devices and Circuits (Ref. 10), p. 124

${ }^{12}$ Charles Kittel, Introduction to Solid State Physics, 6th ed. (Wiley, New York, 1986), p. 259.

${ }^{13}$ Charles P. Poole, Jr. et al., Superconductivity (Academic, San Diego, 1995).

${ }^{14}$ M. Balkanski, in Optical Properties of Solids, edited by F. Abelès (North-Holland, Amsterdam, 1972).

${ }^{15}$ Charles Kittel, Introduction to Solid State Physics (Ref. 12), p. 276.

${ }^{16}$ Pochi Yeh, Optical Waves in Layered Media, Wiley Series in Pure and Applied Optics (Wiley, New York, 1988).

${ }^{17}$ Charles Kittel, Introduction to Solid State Physics (Ref. 12), p. 271. 